\title{
Susceptibilidade de populações de Simulium (Chirostilbia) pertinax Kollar, 1832 (Culicomorpha, Simuliidae) ao temephos e a um formulado à base de Bacillus thuringiensis var. israelensis
}

\author{
Susceptibility of population of Simulium (Chirostilbia) pertinax Kollar, 1832 \\ (Culicomorpha, Simuliidae) to temephos and Bacillus thuringiensis var. israelensis \\ formulation.
}

\section{Carlos Fernando S. de Andrade*, Armando Castello Branco Jr.**}

\begin{abstract}
ANDRADE, C.F.S. de \& CASTELLO BRANCO Jr., A. Susceptibilidade de populações de Simulium (Chirostilbia) pertinax Kollar, 1832 (Culicomorpha, Simuliidae) ao temephos e a um formulado à base de Bacillus thuringiensis var. isralensis. Rev. Saúde públ., S. Paulo, 25: 367-70, 1991. O uso de pequenas calhas de madeira no leito de riachos, manualmente colonizadas com borrachudos, é proposto para avaliações da eficiência de larvicidas 3 ou 4 horas após as aplicações. A susceptibilidade larval também foi avaliada pelo critério de $\mathrm{TL}_{50}$. Em três ensaios no litoral do Estado de São Paulo e Rio de Janeiro, populações de Simulium (C.) pertinax Kollar, 1832 mostraram-se resistentes ao temephos, mesmo em altas concentrações. Vectobac 12 AS, à base de Bacillus thuringiensis var. israelensis mostrou-se mais potente contra larvas dos últimos estádios e eficiente para controle em concentrações a partir de 7.200 UIP/ (10 min.). O TL so $_{0}$ para $3.744 \mathrm{UIP} / 1$ (10 min.) foi de 70,9 minutos.
\end{abstract}

Descritores: Controle de mosquitos. Simuliidae. Bacillus thuringiensis, patogenicidade. Cianamida, toxicidade.

O controle de borrachudos, tanto das espécies transmissoras de doenças como das populações incômodas pela densidade do seu ataque, tem sempre se constituído em grande desafio na área de saúde pública.

Devido ao fato das populações precisarem ser controladas durante normalmente $o$ ano todo, com aplicações periódicas de inseticidas e em vastas áreas, são vários os casos onde ocorreu o desenvolvimento de resistência, desde os primeiros registros no Japão, em 1963, até os relatos do Programa de Controle de Oncocercose, na África ${ }^{9.16,17}$. O inseticida químico temephos é empregado rotineiramente há vários anos no Brasil, e o desenvolvimento de resistência das larvas de borrachudos a esse ingrediente ativo é hoje uma realidade no nossoo país ${ }^{1,14,22}$. A possibilidade dessa resistência ocorrer mesmo em áreas onde esse princípio ativo nunca foi empregado, seja pela migração de indivíduos de populações resistentes, seja pela resistência cruzada a outros organofosforados, toma a sua detecção obrigatória ao se decidir pelo uso desse larvicida². Quando seu uso não é mais possível, a melhor opção tem sido o controle biológico por

* Departamento de Zoologia do Instituto de Biologia da Universidade Estadual de Campinas. Campinas, SPBrasil.

** Pós-graduando em Ecologia do Instituto de Biologia da Universidade Estadual de Campinas. Campinas, SP-Brasil.

Separatas/Reprints: C.F.S. de Andrade .. Caixa Postal 6109 - 13081 - Campinas, SP, Brasil.

Publicação financiada pela FAPESP. Processo 90/4602-1 produtos à base de Bacillus thuringiensis var. israelensis (Bti). Tal medida já vem sendo adotada desde 1980 no programa africano de controle da oncocercose" $e$ há alguns anos no Brasil, no Estado do Rio Grande do $\mathrm{Sul}^{21}$ e, mais recentemente, no litoral norte do Estado de São Paulo ${ }^{4}$.

A eficiência dos tratamentos por Bti está intimamente relacionada tanto a fatores bióticos, tal como a susceptibilidade da população alvo, quanto aos abióticos, como a formulação empregada e as características do curso d'água criadouro. Nesse sentido, as avaliaçõees de eficiência são mais realistas se desenvolvidas no campo, sob condições semelhantes às do criadouro natural.

O presente trabalho se propõe a avaliar a susceptibilidade de larvas de $S$. (C.) pertinax ao temephos e a um formulado comercial à base de Bti, utilizando-se um sistema de calhas de madeira entre as pedras do riacho onde elas se criam. Tais avaliações visam acrescentar ainda conhecimentos aos programas oficiais de controle dessa espécie antropofílica.

\section{Material e método}

Os ensaios foram realizados entre janeiro e março de 1987 nos municípios de Ubatuba e Ilhabela no litoral norte do Estado de São Paulo, dentro de uma vasta área sujeita ao controle periódico de borrachudos pela Superintendência de Controle de Endemias (SUCEN), e no Município de Paraty, ao sul do Estado do Rio de Janeiro, em área não sujeita a controle.

Calhas de madeira de $60 \mathrm{~cm}$ de comprimento por $15 \mathrm{~cm}$ de largura e com bordas de $5 \mathrm{~cm}$ de altura eram dispostas entre as pedras do riacho próximas aos 
trechos criadouros de $S .(C$.) pertinax. Seu arranjo era feito de forma a receberem uma quantidade de água que permitisse velocidades semelhantes à da região do riacho onde estavam. Sua extremidade posterior (de saída) ficava alta, permitindo o cálculo de vazão avaliando-se o volume de água coletado em um balde durante um tempo determinado. As calhas eram forradas com um tecido de algodão branco, para facilitar a fixação das larvas e sua visualização.

As larvas foram coletadas em locais adjacentes às calhas, passando-se um pano nas pedras onde se encontravam fixadas. Desse pano elas eram transferidas para as calhas onde prontamente se fixavam graças ao fio de seda que produzem e ao disco de ganchos no final do abdome. Larvas prejudicadas pelo método de coleta, não se fixavam nas rampas.

Os produtos avaliados foram o Vectobac 12 AS (Abbott Lab.); um formulado de suspensão aquosa com 1.200 unidades internacionais de potência (UIP) por miligrama e o Abate 500 E (American Cyanamid), com 50\% do ingrediente ativo temephos.

No primeiro experimento, foi verificado o Tempo Letal Mediano $\left(\mathrm{TL}_{50}\right)$ e a eficiência em termos da percentagem final de mortalidade ( $3 \mathrm{~h}$ após) para a concentração de $3.744 \mathrm{UIP} / 1$ (durante $10 \mathrm{~min}$ ) para o produto microbiano. Para o larvicida químico, suspeitando-se da resistência, foi avaliada uma concentração alta; de $2,5 \mathrm{mg}$ i.a./l (10 min). A população constituía-se apenas de larvas grandes (histoblastos bem desenvolvidos) de $S$. $(C$.) pertinax sendo colonizadas 3 calhas com 100 indivíduos em cada uma. O ensaio foi no Rio Promirim (Ubatuba), em área rotineiramente tratada pela SUCEN, com temephos.

No segundo experimento, as mesmas concentrações foram avaliadas em termos de eficiência $(3 \mathrm{~h}$ após), numa população mista de $S$. (C. ) pertinax (48\%), S. (C. )spinibranchium Ramirez P., 1982 (40\%) e S. (Tyrsopelma) scutistriatum Lutz, 1910 (12\%). Esse ensaio foi montado no Riacho Independência (Paraty), a $6 \mathrm{Km}$ da divisa com o Estado de São Paulo e portanto, em área não sujeita às aplicações periódicas de temephos. As larvas foram categorizadas em pequenas, sem histoblasto aparente; médias, com histoblasto ainda não diferenciado; e em larvas grandes dos últimos estádios, com o histoblasto já diferenciado em filamentos branquiais. Foram colonizadas 3 calhas com 346 larvas em média em cada uma.

Em outro experimento, apenas o temephos foi avaliado contra larvas grandes de $S$. (C.) pertinax, nas concentrações de 0,5 e $2,5 \mathrm{mg} /(10 \mathrm{~min})$. Usouse respectivamente 54 e 79 larvas. Esse terceiro ensaio foi feito no Ribeirão da Lage, na ponta sul da Ilha de São Sebastião, local não tratado pela SUCEN, na época, e distante a $4,7 \mathrm{Km}$ além do mais próximo curso d'água tratado.
Nos três primeiros experimentos a vazão nas calhas foi calculada com o auxílio do balde, sendo que as aplicações foram feitas gotejando-se os produtos na região superior destas.

No quarto ensaio, realizado na Baía de Castelhanos/Ilhabela, a vazão foi calculada para o riacho todo, considerando-se a relação entre a velocidade da água e o tamanho médio do riacho no trecho avaliado, usando-se a fórmula proposta por Leitritz ${ }^{18}$. Quatro calhas foram dispostas em série no riacho, distando cerca de $10 \mathrm{~m}$ entre si. Foi avaliado em termos de percentagem final de mortalidade ( $4 \mathrm{~h}$ após) apenas Bti, em 4 concentrações va-riando de 792 a 21.600 UIP/l (10 min). Essas concentrações foram estabelecidas em sequiência crescente em relação ao curso d'água, e os tratamentos foram feitos entre as calhas, de modo que a sobreposição das aplicaçõess acima de cada calha permitia a concentração final desejada. A população era mista em igual proporção, de larvas grandes de $S$. (C.) pertinax e $S .(C$.) distinctum Lutz, 1910, e foram usados 75 indivíduos por tratamento.

Em todos os casos, um período de no mínimo 30 min era aguardado, antes dos tratamentos, para permitir a ambientação das larvas. A cada leitura, as larvas mortas ou com a paralisia irreversível eram reconhecidas por não reagirem à aproximação da pinça e retiradas das calhas.

$\mathrm{O} \mathrm{TL}_{50}$ foi calculado pelo método de regressão linear entre o próbite da percentegem de mortalidade e o logarítimo do tempo, usando-se um programa escrito em BASIC, para microcomputador.

\section{Resultados}

O curto período de tempo para leitura das mortalidades finais, de 3 ou $4 \mathrm{~h}$ após as aplicações, permitiu o não desprendimento das larvas mortas e a não colonização de larvas a partir de regiões no riacho acima das calhas.

No primeiro experimento a água caracterizava-se por apresentar temperatura média de $20,0^{\circ} \mathrm{C}, \mathrm{pH}=6,0$ e vazão nas calhas de $132 \mathrm{l} / \mathrm{min}$. $\mathrm{O} \mathrm{TL}_{50}$ obtido para o Bti foi de $70,9 \mathrm{~min}$, com intervalo de confiança (P $<0,05$ ) entre 60,65 e $82,88 \mathrm{~min}$ (Figura), e a mortalidade final após $3 \mathrm{~h}$ da aplicação foi de $85,5 \%$. Para a aplicação do temephos não houve mortalidade alguma até o final das observações.

No segundo experimento a água caracterizava-se por temperatura média de $17,5^{\circ} \mathrm{C}$ e $\mathrm{pH}=6,0$. A vazão média nas rampas era de $68,7 \mathrm{l} / \mathrm{min}$, permitindo uma velocidade comparável à do criadouro e praticamente metade da estabelecida no primeiro experimento. A Tabela apresenta as percentagens finais de mortalidade obtidas para os dois larvicidas após $3 \mathrm{~h}$ da aplicação. 


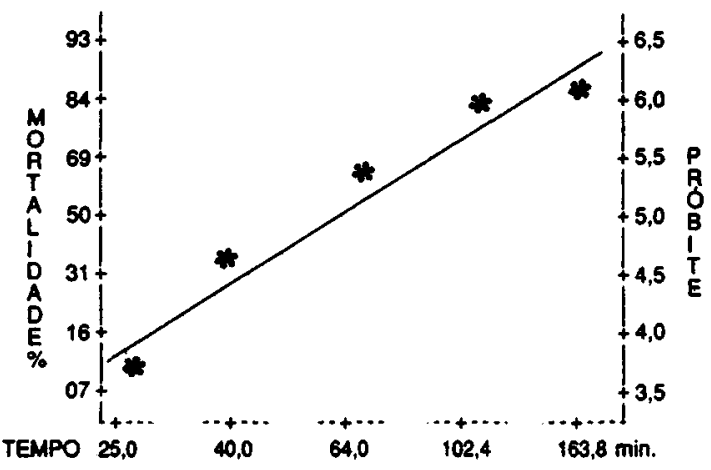

Figura. Regressão tempo-mortalidade para larvas de Simulium (C.) pertinax tratadas com a concentração de 3.744 UIP/l (10 min) de Bacillus thuringiensis var. israelensis.

Tabela. Eficiência 3 horas após a aplicação de Bacillus thuringiensis var. israelensis e temephos contra larvas de três idades de $S$. (C. ) pertinax, $S$. (C.) spinibranchium $e$ S. (T.) scutistriatum.

\begin{tabular}{lrrrrr}
\hline \multicolumn{2}{c}{$\begin{array}{c}\text { Concentração/ } \\
\Lambda(10 \mathrm{~min})\end{array}$} & \multicolumn{4}{c}{ \% de mortalidade em larvas } \\
& Pequenas & Médias & Grandes & Geral \\
\hline Bti & $3.744 \mathrm{UIP}$ & 100 & 93,0 & 81,48 & 90,43 \\
Temephos $2,5 \mathrm{mg}$ & 5,26 & 4,41 & 4,90 & 4,89 \\
\hline
\end{tabular}

No terceiro experimento as calhas foram estabelecidas com vazões de $90 \mathrm{l} / \mathrm{min}$. A temperatura média era de $20,0^{\circ} \mathrm{C}$ e o $\mathrm{pH}=6,0$. Não foram observadas qualquer mortalidade ou desprendimento de larvas vivas para as duas concentrações de temephos avaliadas até o final das observações.

No último experimento a água do riacho encontrava-se com temperatura média de $21^{\circ} \mathrm{C}$ e $\mathrm{pH}=6,0$. A vazão, nesse caso calculada para todo o curso d'água, era de $5,27 \mathrm{~m}^{3} / \mathrm{min}$. As taxas de mortalidade final obtidas para as quatro concentrações usadas do Bti, foram: 80,$6 ; 88,4 ; 100$ e $100 \%$, resectivamente para 792, 2.400, 7.200 e 21.600 UIP/ (10 min).

\section{Discussão}

Os resultados obtidos no primeiro experimento em termos da $\mathrm{TL}_{s 0}$, indicam uma alta susceptibilidade das larvas de $S$. $(C$. ) pertinax ao produto microbiano, quando comparado com os obtidos por Habib's para $S$. goeldi e S. rorotaensis, a despeito de ter havido $14,5 \%$ de sobrevivência $3 \mathrm{~h}$ após o tratamento. A concentração utlizada corresponde a 3,12 ppm do produto comercial, e as recomendadas pelo fabricante situam-se entre 0,5 e $25 \mathrm{ppm}$. Avaliações 12, 24 ou mesmo $48 \mathrm{~h}$ após os tratamentos, como é adotado por vários autores, poderiam resultar em mortalidades maiores, no entanto irreais, pois, na prática, larvas submetidas às concentraçōes letais totais $\left(\mathrm{CL}_{100}\right)$ podem morrer em 15 ou 20 min após o início dos tratamentos, e portanto 3 ou no máximo 4 horas seriam suficientes para toda a expressão do efeito larvicida ${ }^{12}$. A estabilização da mortalidade, que aos $163 \mathrm{~min}$ era de $84 \%$ (Figura) e aos $180 \mathrm{~min}$ $85,5 \%$ evidencia tal conclusão.

A ausência total de mortalidade no tratamento com temephos confirma 0 alto grau de resistência suspeito nessa população. A concentração experimentada foi intencionalmente alta, e equivale a cerca de 17 vezes a concentração usualmente empregada no Brasil para controles.

Os resultados do segundo ensaio indicam para aquela população mista uma susceptibilidade ao bacilo bastante comparável à encontrada só para $S$. (C.) pertinax no primeiro experimento, considerando-se que nesse caso as larvas grandes apresentaram $81,48 \%$ de mortalidade final.

Pode-se verificar ainda, que as larvas mais jovens foram mais susceptíveis à bactéria, fato também apontado por outros autores ${ }^{8.10,12.19}$. A pequena mortalidade causada pelo temephos, nessa concentração alta e ainda em área não sujeita ao controle periódico, só poderia ser explicada devido a um baixo grau de isolamento entre essa população e as adjacentes, da área sob controle distante menos de $10 \mathrm{Km}$. Uma alta pressão de seleção, com tratamentos durante o ano todo, a curtos intervalos (15 a 22 dias), em vasta área $\left(890 \mathrm{Km}^{2}\right)$ e durante vários anos (desde 1971), devem ter levado à dominância os genes de resistência em complexos gênicos agora adaptados ao ambiente. A migração de indivíduos assim resistentes levaria as populações vizinhas a fixarẹm também a resistência, embora nunca tenham sido expostas diretamente ao inseticida. Esses fatores têm sido abordados por alguns autores ${ }^{6,7,20} \mathrm{e}$ igualmente explicam a resistência ao temephos detectada na população do terceiro ensaio (Ribeirão da Lage).

A população mista avaliada no último ensaio, submetida a concentrações calculadas para todo o riacho, mostrou uma susceptibilidade semelhante àquelas anteriormente detectadas (Paraty e Ubatuba). Pode-se sugerir, assim, o uso dessa formulação em concentrações não inferiores a 7.200 UIP/l (10 min). Ruas Neto ${ }^{21}$, em uma série de aplicações de campo, indica que essa mesma concentração (mesmo formulado), em aplicaçõess durante um minuto, causaram entre 87 e $100 \%$ de eficiência ( $24 \mathrm{~h}$ após) em larvas de $S$. (C. ) pertinax no Rio Grande do Sul. Outros autores ${ }^{3,13}$ relatam ainda a eficiência do Bti contra $S$. (C. ) pertinax no Brasil, no entanto a não citação da formulação utilizada ou mesmo de sua potência nessas poucas referências, dificulta ou mesmo impossibilita comparaçōes.

Os programas de controle de borrachudos desenvolvidos no Brasil seja por órgãos públicos ou pela 
iniciativa privada, sofrem diferentes graus de dificuldade pela falta de conhecimentos bioecológicos das espécies alvo e da eficiência dos agentes de controle. Ensaios de campo, como os desenvolvidos no presente trabalho, podem ser feitos em um dia por 2 ou 3 pessoas, permitindo a verificação da eficiência de larvicidas sob condições naturais, passo importante para o estabelecimento de programas de controle e para o seu monitoramento.

ANDRADE, C.F.S. de \& CASTELLO BRANCO Jr., A. Susceptibility of population of Simulium (Chirostilbia) pertinax Kollar, 1832 (Culicomorpha, Simuliidae) to temephos and Bacillus thuringiensis var. israelensis formulation. Rev. Saúde públ., S. Paulo, 25: 367-70, 1991. The use of wooden troughs on stream beds, artificially colonized by blackfly larvae, is proposed for larvicide evaluations. Mortality was recorded 3 or 4 hours after treatment. Larval susceptibility was also evaluated utilizing the $\mathrm{LT}_{50}$ criterion. In there field assays Simulium $(C$.) pertinax populations from the litoral of $S$. Paulo and Rio de Janeiro States were shown to be resistant to temephos, even when subjected to high concentrations. Vectobac 12 AS, a Bacillus thuringiensis var. israelensis product, was shown to be more potent against late instar larvae and efficient in concentrations higher than 7,200 ITU/1 (10 $\mathrm{min})$. The $\mathrm{LT}_{50}$ to $3,744 \mathrm{ITU} / \mathrm{h}(10 \mathrm{~min})$ was calculated as $70.9 \mathrm{~min}$

Keywords: Simuliidae. Bacillus thuringiensis, pathogenicity. Cyanamide, toxicity. Mosquito control.

\section{Referências Bibliográficas}

1. ANDRADE, C.F.S. Resistência de populações de simulídeos à inseticidas. In: Seminário Nacionall de Vetores Utanos e Animais Sinantrópicos, 2\%/Reunião Brasileira sobre Simulídeos, $3^{2}$, Porto Alegre, 1988. Resumos. Porto Alegre 1988. p. $59-60$

2. ANDRADE, C.F.S. \& CASTELLO BRANCO JR., A. Methods for field detection of resistance to temephos in simuliids: larval esterase level and topical application of the insecticide to adults. Mem. Inst. Oswaldo Cruz, Rio de Janeiro, 85: 291-8, 1990.

3. ARAUJO-COUTINHO, C.J.P.C. \& LACEY, L.A. Avaliação em condições naturais de três concentrados emulsionáveis de Bacillus thuringiensis var. israelensis (H-14) para o controle de simulídeos no Litoral Norte do Estado de São Paulo. In: Serminário Nacional de Vetores Urbanos e Animais Sinantrópicos, 1․ São Paulo, 1986. Resumos. São Paulo, 1986. p. 27

4. ARAUJO-COUTINHO, C.J.P.C. \& LACEY, L.A. Controle de simulídeos com concentrado emulsionável de Bacillus thuringiensis. Bol. Ofic. sanit. panamer., 108: 213-9, 1990.

5. CYANAMID. Agricultural Research Division. Abate-Larvicida. Princeton, N.J., American Cyanamid Co., 1980.

6. COMINGS, H.N. The development of insecticide resistance in the presence of migration. J. Theor. Biol., 64: 177-97, 1977.

7. CURTIS, C.F.; COOK, L.M.; WOOD, R.J. Selection for and against insecticide resistance and possible methods of inhibiting the evolution of resistance in mosquitoes. Ecol. Entomol, 3: 273-87, 1978

8. GUILLET, P. \& BARJAC, H. de. Toxicité de Bacillus thuringiensis var. israelensis pour les larves de simulies vectrices de l' onchocercose. C. R. Acad. Sci. Paris, 289 D.: 549-52, 1979.

9. GUILLET, P.; ESCAFFRE, H.; OUEDRAOGO, M. QUILÉVÉRÉ, D. Mise en evidence d'une résistance au téméphos dans le complexe Simulium damnosum ( $S$. sanctipauli et $S$. soubrense) en Côte D'Ivoire. (Zone du Programme de lutte contre l'Onchocercose dans Région du Bassin de la Volta). Cah. ORSTOM. Ser. Ent. méd. parasit. 17: $291-9,1980$.

10. GUILLET, P.; ESCAFFRE, H.; PRUD'HOM, J-M. L'utilisation d'une fomulation à base de Bacillus thuringiensis H14 dans la lutte contre l' onchocercose en Afrique de l'Ouest. 1. Efficacité et modalités d'aplication. Cah. ORSTOM. Ser Ent. méd. parasit., 20: 175-80, 1982.

11. GUILLET, P.; ESCAFFRE, H.; PROD'HOM, J-M. BAKAYOKO, S. Étude des facteurs conditionnant l'efficacité des préparations à base de Bacillus thuringiensis H-14 vis-à-vis des larves - du complexe Simulium damnosum (Diptera, Simuliidae). 1. Influence de la nature e de la taille des particules. Cah. ORSTOM. Sér. Ent. méd. parasit., 23: 257-64, 1985

12. GUILLET, P.; HEUGARD, J-M; DOANNIO,J.; ESCAFFRE, H.; DUVAL, J. Evaluation de la sensibilité des larves du complexe Simulium damnosum à la toxine de Bacillus thuringiensis H-14. 1. Metho dologie. Cah. ORSTOM. Sér. Ent. méd. Parasit., 23: 241-50, 1985.

13. GUIMARÃES, E.L.G. Eficiência de Bacillus thuringiensis var. istaelensis De Barjac no controle de larvas de borrachudos. (Diptera: Simuliidae). In: Congresso Brasileiro de Zoologia, $12^{\circ}$, Campinas, SP, 1985. Resumos. Campinas, 1985. p. 123.

14. GUIMARÃES, E.L.G. Biologia e controle de simulídeos no Estado do Paraná. In: Seminário Nacinal de Vetores Urbanos e Animais Sinantrópicos, $1^{\circ}$, São Paulo, 1986. Resumos. São Paulo, 1986. p. 24

15. HABIB, M.E.M. Potency of Bacillus thuringiensis var. israelensis ( $\mathrm{H}: 14)$ against some aquatic dipterous insects. $Z$. ang. Ent., 95: 368-76, 1983.

16. KURTAK, D. Insecticide resistance in the Onchocerciasis Control Programme. Parasit. Today, 2: 19-20, 1986.

17. LAIRD, M. \& MILES, J.W. Integrated mosquito control methodologies: experience and components fiom convencional chemical control. New York, Academic Press, 1983. v.1

18. LEITRITZ, E. Trout and salmon culture. Fish Bull., (107) 1959.

19. MOLLOY, D.; GAUGLER, R.; JAMNBACK, H. Factors influencing efficacy of Bacillus thuringiensis var. israelensis as a biological control agent of black fly larvae. J. Econ. Ent. 74: $61-4,1981$.

20. ORGANIZACION MUNDIAL DE LA SALUD. Comite de Expertos en Insecticidas, Ginebra, 1975. Resistencia de vectores y reservorios de enfermedades a los plaguicidas; $22^{\circ}$ informe. Ginebra, 1976. (Série de Informes Técnicos, 585)

21. RUAS NETO, A.L. Bacillus thuringiensis var. istaelensis como altemativa no controle de simulídeos no Rio Grande do Sul. 1 - Susceptibilidade a campo. BS Bol. Saúde, Porto Alegre, 11: $21-6,1984$.

22. RUAS NETO, A.L. Avaliaçāo do uso de temephos para o controle de simulideos no Rio Grande do Sul. BS Bol. Saúde. Porto Alegre, 11: 27-31, 1984.

Recebido para publicaçāo em 12/11/1990 Reapresentado em 17/6/1991 Aprovado para publicação em 25/6/199/ 